\title{
Ensino baseado em problemas como prática pedagógica aplicada a alunos ingressantes no curso de Odontologia
}

\author{
Magali Luci Pinto*, Florence Zumbaio Mistro**, Sofia Takeda Uemura ${ }^{* * *}$
}

* Tutora do PBL, Professora do Centro Universitário Hermínio Ometto, FHO Uniararas.

** Professora da área Clínica e Estomatologia do Centro Universitário Hermínio Ometto, FHO Uniararas.

*** Coordenadora da área de Odontologia para Pacientes Especiais do Centro Universitário Hermínio Ometto, FHO Uniararas

\begin{abstract}
RESUMO
A dificuldade apresentada pelos profissionais da área da saúde quando no início do exercício de sua profissão tem sido amenizada com o uso de práticas metodológicas diferenciadas durante a sua formação, como o ABP (Aprendizado Baseado em Problemas) mais conhecido como Problem Based Learning (PBL). O presente estudo relata a experiência do uso do PBL como prática de extensão para os alunos ingressantes no curso de Odontologia. Casos clínicos reais e hipotéticos são apresentados com o objetivo de resgatar o conteúdo da estrutura curricular e acrescentar gradualmente o uso de termos específicos da área e o desenvolvimento da capacidade de avaliar condutas. Cada caso foi apresentado em duas sessões, a partir das quais cada grupo elaborava um diagnóstico, identificava os conceitos multidisciplinares presentes e os a serem adquiridos. Com o decorrer da prática, as discussões dos casos e propostas de diagnósticos foram realizadas como reuniões clínicas para prática de liderança e interação de equipe. Concluímos que a prática pedagógica PBL, utilizada desde o início do curso, contribui para a formação de um estudante com perfil diferenciado, mais capacitado ao aprendizado de disciplinas específicas e mais seguro para atuação no mercado de trabalho.
\end{abstract}

Descritores: Aprendizagem Baseada em Problemas. Práticas Pedagógicas. Odontologia. 


\section{INTRODUÇÃO}

Veiga ${ }^{1}$ defende que as práticas pedagógicas devem ser fundamentadas como uma prática social orientada por objetivos, finalidades e conhecimentos inseridos no contexto da prática social, apresentando uma relação teórico-prática, afirmando que é nosso dever como educadores a busca de condições necessárias para sua realização.

Norteados por esse conceito, as práticas pedagógicas aqui são tratadas como um conjunto de atividades planejadas e desenvolvidas no âmbito escolar, vinculadas à realidade social $\mathrm{e}$ cultural dos seus aprendizes e, portanto, assumem uma dimensão pedagógica não só educativa e sim interativa no contexto da dinâmica social para realização do aprendizado.

Dentre as diferentes práticas pedagógicas inovadoras e tradicionais utilizadas em vários cursos de graduação, o PBL (Problem Based Learning) ou também chamado de ABP (Aprendizagem Baseada em Problemas) foi escolhido para ser aplicado aos alunos ingressantes no curso de Odontologia do Centro Universitário Hermínio Ometto (FHO Uniararas).

O PBL objetiva preparar o aluno para atuar profissionalmente de forma diferenciada, trazendo para a sala de aula situações representativas de problemas reais que ele irá vivenciar na profissão. Fundamenta-se como um processo de educação continuada como meio de construção de conhecimento que deve ocorrer desde o início do curso com o perfil de uma aprendizagem autodirigida $^{2}$.

Outro ponto crucial dessa prática refere-se à interdisciplinaridade da estrutura curricular. $\mathrm{O}$ discente precisa resgatar conhecimentos prévios de várias áreas que já foram desenvolvidas em sala de aula, para interpretar uma situação apresentada e assim propor soluções.

Atualmente, um dos grandes problemas enfrentados por várias universidades é desenvolver uma proposta curricular que consiga transmitir conceitos gerais e específicos a uma população acadêmica que carrega formações e vivências sociais que muitas vezes entram em conflito com o perfil da universidade, gerando o desestímulo e a desistência.

Não podemos também esquecer as mudanças cognitivas e emocionais vivenciadas pelo estudante durante a graduação. Muitos são bastante jovens e o grau de maturidade emocional reflete significativamente no seu aprendizado ${ }^{3}$.

Durante algum tempo a formação puramente acadêmica era a única exigência para adentrar no mercado de trabalho e ali permanecer até aposentadoria ou morte. Atualmente, a nossa realidade acadêmica inserida em um avanço tecnológico e de pesquisas, exige outras habilidades. Desta forma, o papel do conhecimento de formação tem que ser renovado constantemente.

O objetivo do presente artigo é relatar a experiência da aplicação do PBL com discentes do primeiro ano do curso de Odontologia como atividade de extensão.

\section{RELATO DE EXPERIÊNCIA}

Os casos clínicos e ou situações reais foram elaborados para contemplar o conteúdo programático da estrutura curricular do curso de Odontologia tanto no período integral como no noturno.

O PBL foi desenvolvido em dois encontros de modo que no primeiro era apresentado o caso e no segundo, realizado o encerramento. Barrows ${ }^{4}$ (1986) descreveu em seis passos como devem ocorrer as sessões do PBL, o que serviu para elaborar pedagogicamente a evolução da sessão tutorial (quadro 1).

Previamente à aplicação da prática pedagógica, vale ressaltar que no primeiro dia os alunos são orientados sobre como se dará cada sessão do PBL. Assim, na aula inaugural, o tutor apresenta o contexto do PBL, ressaltando seus objetivos e sua aplicabilidade e em seguida expõe como será desenvolvido em cada encontro.

Para avaliar se o PBL contemplava e atingia seus objetivos no curso de Odontologia da mesma forma que tem se mostrado em outros cursos, 
Quadro 1 - Estrutura das sessões do PBL.

\begin{tabular}{|l|l|}
\hline Primeira Sessão & Segunda Sessão \\
\hline Apresentar o caso & $\begin{array}{l}\text { Leitura oral pelo coordenador } \\
\text { Descrição da conduta do grupo }\end{array}$ \\
\hline Escolher coordenador/assistente & $\begin{array}{l}\text { Identificar no caso a estrutura curricular } \\
\text { Apresentação do diagnóstico do grupo pelo coordenador }\end{array}$ \\
\hline Leitura oral pelo coordenador & Discussão entre os coordenadores \\
\hline Identificação de termos desconhecidos & Comentários e correções pelo tutor \\
\hline Discussão entre o grupo & Fechamento do caso com o diagnóstico e conduta a ser tomada \\
\hline Listar conteúdo a ser estudado & \\
\hline
\end{tabular}

(Medicina, Enfermagem e Engenharia), duas avaliações foram realizadas, conforme descrito a seguir:

- o tutor tem uma ficha para avaliação individual do discente no início e ao término do caso, tendo como parâmetros a presença, participação e postura; e

- uma avaliação semestral que aborda um caso clínico para ser interpretado individualmente é aplicada.

Embora o PBL tenha sido aplicado como atividade de extensão, optamos por dar ao aluno no final do semestre o certificado constando as horas cumpridas e a nota final obtida nas avaliações. Portanto, conceitos foram adotados, onde a avaliação individual tem um peso de zero até 0,4 e a avaliação escrita de zero a 0,6.

\section{Caso 1 - Acidente}

Acidente na segunda-feira da semana passada em Villa Castelli deixou a França em estado de choque. As duas aeronaves se chocaram na pequena cidade de Villa Castelli, 1.400 quilômetros a noroeste de Buenos Aires, e os tanques de combustível explodiram no acidente. A tragédia matou os dois pilotos argentinos, cinco esportistas franceses e três esportistas brasileiros: a velejadora veterana Fernanda Arthaud, a nadadora Camille Mendonça, campeã olímpica em Londres/2012 e o boxeador Alexandre Veiga, medalhista de bronze em Peq- uim/2008. O acidente também matou cinco membros da produção do programa de televisão "Dropped", transmitido pelo canal TF1. "Com a colaboração dos cirurgiões-dentistas da equipe, terminamos o trabalho de campo e iniciamos a fase de análise laboratorial em colaboração com o Escritório de Investigação e Análise para a Segurança da Aviação Civil (BEA) da França”, indicou em um comunicado a Junta de Investigação de Acidentes da Aviação Civil (JIAAC). Os restos dos passageiros brasileiros foram devolvidos às suas famílias.

Sua equipe é convocada e se dirige ao IML da cidade de Bueno Aires. No local, sobre a bancada, estão as calotas cranianas e mandíbulas.

Objetivos propostos: Quais as disciplinas precisam ser resgatadas? Descreva toda a postura que a sua equipe precisa ter. Quais ferramentas foram utilizadas para identificar os três brasileiros?

\section{Caso 2 - Mucocele}

Paciente do gênero feminino, 40 anos de idade, compareceu à triagem da clínica odontológica manifestando o desejo de tratamento de seus dentes e, se possível, a instalação de uma prótese dentária, alegando dificuldades na mastigação pela ausência de dentes posteriores e inferiores. $\mathrm{O}$ aluno Carlos Henrique, sob a supervisão do docente de clínica, inicia a anamnese. A paciente relata ausência de problemas de saúde e de sintomatologia. O aluno 
inicia o exame físico extra e intraoral, observa a presença de um aumento de volume (aproximadamente $5 \mathrm{~mm}$ de diâmetro) na região do lábio inferior, indolor, flutuante, bolhoso e translúcido, sugerindo mucocele.

Após o atendimento, a paciente é agendada para uma próxima sessão, possibilitando assim o planejamento do caso.

Objetivos propostos: terminologia usada; conceituar anamnese; diferenciar exame físico extraoral e intraoral; localização anatômica; investigação à respeito do mucocele (etiopatogenia, diagnóstico e tratamento); qual deve ser a orientação prescrita pelo aluno à paciente e indicação de fontes de pesquisa.

O tutor deve ressaltar para os grupos a importância de ser adotada uma sequência para realização do PBL, mencionadas no quadro 1 e, que essa conduta frente a um caso clínico com o passar do tempo será realizada automaticamente pelos mesmos e irá fazer parte de sua conduta profissional.

Após o período de uma semana, todos se reunem novamente com o objetivo de fechar o caso.

Existem alguns pontos a serem considerados pelo tutor nesse segundo encontro, tais como: ausência de algum integrante do grupo; ausência do coordenador do caso em vigência; a não participação de algum integrante do grupo; a postura que foi adotada pelo coordenador quando o grupo é divergente.

Toda e qualquer conduta tomada pelo tutor frente aos imprevistos acima citados, deverá ser passada a todos os alunos no início da prática do PBL. Esta é uma etapa muito importante, o tutor consegue trabalhar nesse momento o que há de mais importante no projeto que é o comprometimento do aluno dentro de uma equipe e frente a uma situação. Neste momento, atitudes disciplinares são fundamentais. Deve estar claro para o estudante o quanto a sua postura durante as sessões vai interferir no trabalho final da equipe.

Ao término de cada caso é conveniente que o tutor converse com cada grupo separadamente, abordando os aspectos positivos e negativos que ocorreram durante o caso e proponha possíveis mudanças. Alguns aspectos são fundamentais para serem observados e discutidos como: pontualidade, assiduidade, perfil comportamental, relacionamento entre o grupo, relacionamento social, desenvolvimento de raciocínio clínico, análise crítica, construção do conhecimento, integração de conhecimentos, rendimento e produtividade de tarefas, análise de fontes de consulta e liderança.

Outro aspecto importante que deve ser analisado pela instituição é o perfil do docente que atuará como tutor, principalmente em relação à sua integração com os demais docentes.

Participaram da aula inaugural do projeto 70 alunos do curso de Odontologia do período integral e noturno, quando foi realizado a explanação do PBL e como seria desenvolvido. Todos os aspectos metodológicos do PBL foram cumpridos.

$\mathrm{Na}$ segunda sessão, realizada após o intervalo de uma semana, estavam presentes 72 estudantes, embora dois deles tenham faltado na abertura do caso, participaram do estudo e não demonstraram dificuldades, pois foram orientados pelos coordenadores dos grupos aos quais se juntaram.

Durante a discussão dos casos notou-se que no grupo de coordenadores havia discentes com mais desenvoltura e firmeza. Outros, com grande dificuldade de se expressar por timidez. Observouse, ainda, o uso de "gírias" que fazem parte da linguagem estudantil.

Durante as discussões e apresentações foi trabalhada a terminologia específica da Odontologia, identificação das disciplinas envolvidas no caso, postura, uso da linguagem acadêmica, fontes de pesquisa, liderança, interação em grupo e diversidade de opiniões.

Os alunos manifestaram sua opinião com respeito ao PBL como metodologia suplementar, com o papel de integração disciplinar. Houve $100 \%$ de aceitação do PBL como prática pedagógica para o curso de Odontologia, 
conforme relatos dos discentes e docentes atuantes no grupo.

\section{DISCUSSÃO}

O avanço das tecnologias de informação e comunicação (TIC) nos surpreende a cada dia, interagindo na vida do homem em vários âmbitos e faixas etárias, conduzindo a todos os expectadores, ativos ou não, educadores ou não, a tomar posição dentro desse novo mundo interativo.

Embora pareça que a ideia de um aprendizado de forma mais ativa esteja vinculado a pesquisas e tecnologias, ela é muito antiga (470399 a.C.). O aprendizado ativo é um pensamento defendido por Sócrates, segundo o qual a busca pelo conhecimento engrandece a alma e o indivíduo. Também em outros tempos, na década de 50, John Dewey ${ }^{5}$, um renomado educador americano, defendia a tese de que "aprender fazendo" faria toda a diferença para o estudante durante e após sua formação educacional ${ }^{6}$.

$\mathrm{O}$ pensamento defendido por Dewey ${ }^{5}$ era compartilhado pelos movimentos estudantis da época, que manifestavam não somente contra a Guerra do Vietnã, mas por mudanças políticas e pedagógicas nas universidades.

Ocorre então no ano de 1969, na universidade Mc Master (Hamilton, Ontário Canadá), a criação de um novo modelo pedagógico. Uma visão pedagógica embasada no aprendizado como um processo contínuo de educação que substituiria por completo as aulas tradicionais com o perfil de transmissão de conhecimento. $\mathrm{O}$ princípio básico seria o contato clínico do aluno em formação desde o seu ingresso na faculdade e não apenas quando fosse fazer os estágios. Pensou-se, em um currículo baseado no estudo de problemas reais com a finalidade de uma aprendizagem autodirigida. Este modelo passou a ser chamado de $\mathrm{PBL}^{7}$.

A visão de que a educação era um processo que necessitava de mudanças não era exclusiva de países mais desenvolvidos, também do nosso país e assim pensadores diferenciados como Paulo
Freire $^{8}$ (1970), que criticava o modelo que chamava de "bancário", onde os professores depositavam conhecimentos na "conta" cerebral do aluno. Defendia o modelo ativo e mostrava seu pensamento com a frase "aprende-se a nadar na piscina e não com as aulas teóricas". O revolucionário modelo de alfabetização para adultos, cujos princípios deveriam ser adotados em qualquer etapa do aprendizado, era o chamado "círculo de cultura", com o coordenador de debates (professor) estimulando os participantes do grupo (estudantes) ao diálogo (aula) para a construção do conhecimento.

Outro aspecto que sempre foi considerado com relação ao aprendizado é como o estudante é avaliado.

O processo "ser avaliado" sempre esteve presente em toda e qualquer atividade humana. Sob o olhar educacional, é obter informações por meio de métodos específicos o quanto o aprendiz reteve do seu aprendizado, o seu desempenho escolar.

Segundo Troncon ${ }^{9}$ (1996), o processo de avaliação principalmente nos estudantes de medicina requer não só quantificar o que foi retido de conhecimento pelo estudante, mas principalmente avaliar as mais complexas habilidades relacionadas ao raciocínio clínico, à forma adequada de aplicação do que foi adquirido na solução de problemas concretos. $\mathrm{O}$ autor ainda afirma que essas habilidades e competências clínicas, cuidados com o paciente, obtenção de uma história clínica e não menos importante conseguir direcionar os familiares, são adquiridas na prática profissional.

É muito comum dentro da sociedade acadêmica, a indagação por parte de alguns docentes, sobre o baixo rendimento de um estudante frente a uma avaliação, colocando em dúvida o que foi transmitido em sala de aula.

O baixo rendimento em muitos casos justifica-se pela adoção inapropriada do estudante, em estudar apenas para obter resultados nas avaliações e não para adquirir conhecimento ${ }^{9}$.

Segundo alguns autores, no século XX, a 
visão do que representa uma avaliação foi se alterando. Em 2002, Perrenoud ${ }^{10}$, define a avaliação sendo um processo contínuo mediador da construção do currículo e está relacionada com a gestão do aprendizado. Vignochi ${ }^{11}(2009)$ diz que a avaliação pode ser realizada de várias formas, o importante é traçar um paralelo entre os resultados e o real aproveitamento do estudante.

Com respeito aos aspectos de avaliação dentro do PBL, alguns estudos defendem que são avaliações progressivas e somativas dos conhecimentos trabalhados em cada semestre e que outros aspectos como habilidades e competências também podem ser avaliados, corrigidos e direcionados. ${ }^{12,11,13}$

Educadores formados em escolas tradicionais necessitam conscientizar-se que a ação do educador vai além de reproduzir informação, pois o estudante atual busca a interatividade com a cobrança de um espaço para reflexão e indagação do que está sendo aprendido. É comum hoje, comentários dentro do ambiente de ensino de algo que se leu, ou se ouviu nos meios de comunicação digital. Embora, o discente precise de uma base para interpretar essas informações, é nesse momento que surge o educador como mediador do processo de ensino e aprendizagem de forma crítica.

Embora o PBL tenha surgido na formação dos alunos do curso de medicina, atualmente tem sido utilizado para auxiliar na formação de outras profissões $^{14}$. Segundo Troncon ${ }^{9}$ (1996), avaliar o profissional da área de saúde é uma das etapas mais relevantes durante todo o processo de formação, visto que deve abranger não só o conhecimento adquirido, mas principalmente as habilidades de ordem emocional e raciocínio frente a inúmeros aspectos da prática profissional.

Isto demonstra que o PBL se aplica além do curso de medicina, pois todos os cursos de saúde atualmente são construídos com a pesquisa e com pessoas, exigindo do profissional a capacidade de interagir, liderar e raciocinar.

Recentemente, foi relatada uma experiência vivenciada pela implantação da metodologia PBL para alunos dos cursos de Engenharia Civil, Engenharia de Produção e Engenharia de Computação durante o período de 2002 a 2008, nas escolas de Engenharia de São Carlos e da Universidade de São Paulo. O estudo relata que estas instituições adotaram o PBL nos cursos de graduação em Engenharia de Produção, Engenharia Civil e Engenharia de Computação. Foi relatado que o PBL foi implantado como uma disciplina paralela à estrutura curricular como Teorias da Administração. O objetivo era levar para os estudantes problemas para que os mesmos apresentassem uma solução. No término, cerca de $90 \%$ dos estudantes afirmaram que o método foi positivo no processo de aprendizagem. $\mathrm{O}$ mesmo foi relatado pelos docentes que atuaram como tutores ${ }^{15}$.

A iniciativa destes educadores em conhecer a fundamentação do PBL e se propor à sua execução como tutor evidencia a visão pedagógica de um modelo construtivo, participativo e ativo de aprendizagem e que a prática do PBL é possível de ser aplicada não somente nas áreas diretamente ligadas à saúde como a Medicina, Biomedicina, Farmácia, Enfermagem, Fisioterapia e tantas outras, mas para qualquer área da graduação inclusive na área das Ciências Exatas e Humanas ${ }^{16}$.

No entanto, é preciso ter a conscientização de que, na formação profissional, o uso do PBL deve estar adequado às particularidades da área de conhecimento, ao grupo de docentes e discentes, à instituição e às diretrizes que regem a educação superior do país.

Não se espera que o estudante alcance "respostas corretas", mas consiga lidar com as informações e reconhecer quais são importantes para o diagnóstico e manejo de uma situação clínica. Assim, no PBL o mais importante é o processo e não a solução em si.

Um dos pontos mais discutidos com relação à prática do $\mathrm{PBL}$ entre os grupos de profissionais responsáveis pela estruturação acadêmica é qual o momento certo para a sua aplicação. Universidades como o Centro Universitário São 
Camilo defendem a ideia de que a sua aplicação para os alunos do curso de Medicina ocorra desde o início, possibilitando que o discente adquira gradualmente os conceitos e crie principalmente $o$ hábito e interesse pelo estudo autodirigido, que seja uma prática paralela a grade curricular.

Outras universidades, como a Universidade Federal de Sergipe, adotam o perfil inserido no PBL para desenvolver disciplinas da estrutura curricular. Defendem que os médicos formados pela instituição sejam capazes de desenvolver ações de prevenção, promoção, proteção e viabilização da saúde nos âmbitos individual e coletivo, perfil que caracteriza o papel ativo do formando quando na sua atuação no mercado e não meramente cumpridor de sua carreira.

Todos os estudos acima citados serviram de parâmetro para implantar a prática do PBL no Centro Universitário Hermínio Ometto.

Os resultados obtidos nesse projeto no curso foram de encontro aos observados em várias instituições de Ensino Superior que instituíram a prática do PBL como um apoio paralelo à estrutura curricular desenvolvida de maneira tradicional, com o uso de vários recursos metodológicos de cada disciplina, as ditas aulas teórico-práticas.

Algumas instituições no Brasil adotaram a prática do $\mathrm{PBL}$ como uma metodologia pedagógica para substituir os métodos tradicionais. Uma experiência ainda não vivenciada pelo nosso grupo, portanto não possuímos embasamento para analisá-la.

O PBL foi utilizado na FHO - Uniararas pela primeira vez no curso de Odontologia com os alunos do $1^{\circ}$ ano do período integral e noturno. No primeiro caso apresentado, os discentes tiveram a oportunidade de resgatar conceitos aprendidos na disciplina de anatomia e acrescer diferenças anatômicas pertinentes ao sexo e ainda, diferenças anatômicas diretamente influenciadas pelas práticas esportivas.

Já no segundo caso, abordamos uma patologia, escolhida por envolver conteúdos trabalhados na disciplina de Biologia Estrutural, com conceitos de histologia básica, patologia básica e fisiologia.

O importante é ressaltar que nos tempos atuais precisamos estar constantemente atentos para realizar nosso papel como educadores. O principal objetivo da prática do PBL é proporcionar ao futuro profissional uma aquisição do conhecimento por meio do construtivismo, independência, hábito de estudo e pesquisa bibliográfica.

\section{CONSIDERAÇÕES FINAIS}

A partir do exposto, podemos constatar que o PBL se fundamenta como uma prática pedagógica para qualquer área do ensino superior, favorece a construção de um conhecimento significativo para o estudante e mostra uma visão moderna e multidisciplinar da entidade de ensino.

Essa metodologia propicia o desenvolvimento de habilidades e posturas frente a diversas situações que irão fazer parte da rotina do discente, que o diferenciará no mercado de trabalho.

$\mathrm{O}$ uso do PBL deve estar adequado às particularidades da área de conhecimento, ao grupo de professores e alunos, à instituição e às diretrizes que regem o Ensino Superior do país.

\section{ABSTRACT \\ Problem based learning as a pedagogical practice apllied to freeschman in Dental course}

The difficulty presented by health professionals when the beginning of the exercise of their profession has been reduced with the use of different methodological practices during their training, as Problem Based Learning, known as PBL. This study adopts the PBL as an extension activity for students entering in the Dental course. Real clinical and hypothetical cases are presented with the aim of rescuing the contents of the curriculum and gradually add the use of specific terms and the development of the ability to manage behavior. The cases were presented in two sessions, where each group elaborated a diagnosis, identify the multidisciplinary concepts present and to be acquired. In the course of practice, cases were 
discussed and diagnoses were proposed, as well as the clinical meetings allowed to practice leadership and team interaction. We conclude that the PBL pedagogical practice, used since the beginning of Dental course, helps to form a student with a different profile, more capable of learning specific subjects and safer to act in the labor market.

Descriptors: Problem Based Learning. Pedagogical Practices. Dentistry.

\section{REFERÊNCIAS}

1. Veiga IPA. A prática pedagógica do professor de didática. Papirus. 1994.

2. Pinto ML. Práticas Pedagógicas no Ensino Superior. A aprendizagem baseada em problemas (ABP) ou Problem Based Learning (PBL) na área da saúde. Copyright 2013;183-201.

3. Rosario P, Núñez JC, Pienda-Gonzalez JA. Cartas de Gervásio ao seu umbigo. $2^{\mathrm{a}}$ ed. Almedina. 2006.

4. Barrows HS. A taxonomy of problem based learning. Med-Educ 1986;20(6):482-6.

5. Dewey J. Democracia e educação: introdução à filosofia da educação. 1959. Disponível em: http://www.dominiopublico.gov.br/download texto/me4677.pdf

6. Dewey J. Reconstrução em filosofia. 1959; $2^{\mathrm{a}}$ ed. Tradução de António Pinto de Carvalho Liberalismo, liberdade e cultura. Nacional. 1970.

7. Dewey J. Escola Nova e Construtivismo: continuidade, descontinuidade e recontextualização. In: Almeida, JS. Estudos sobre a profissão docente. Cultura Acadêmica. 2001.

8. Penaforte J. John Dewey e as raízes filosóficas da aprendizagem baseada em problemas. In: Mamede S, Penaforte J, Schimdt H, Caprara A, Tomaz JB, Sá H. Aprendizagem baseada em problemas: anatomia de uma nova abordagem educacional. Editora Hucitec. 2001:49-78.

9. Albanese MA, Mitchell S. Problem-based learning: a review of literature on its outcomes and implementation issues. Acad Med 2003; 68(1):52-81.

10. Freire P. Pedagogia do oprimido. Rio de Janeiro: Paz e Terra. 1970.

11. Troncon LEA. Avaliação do estudante de medicina. Medicina 1996; 29:429-439.

12. Perrenoud P, Gather TM, De Macedo L, Machado NJ, Allessandrini CD. As competências para Ensinar no Século XXI. A Formação dos Professores e o Desafio da Avaliação. Porto Alegre: Artmed, 2002; 11:33-39.

13. Vignochi CM, Benetti CS, Machado CL, Bezerra MWC. Considerações sobre aprendizagem baseada em problemas na educação da saúde. Rev HCPA 2009; 29(1):45-50.

14. Vasconcelos C. Avaliação: concepção dialética-libertadora do processo de avaliação escolar. São Paulo: Libertad. 1994.

15. Price DA. Tutor training for problem-basedlearning: the experience of a large medical school. In: Scherpbier AJJA, van der Vleuten CPM, Rethans JJ, van der Steeg AFW. Advances in Medical Education. SpringerVerlag: Berlim, 1997.

16. Duch BJ, Groh SE, Allen DE. The power of problem-based learning. Sterling Stylus, 2001.

17. Escrivão Filho E, Ribeiro RLC. Aprendendo com PBL - aprendizagem baseada em problemas: relato de uma experiência em cursos de engenharia da EESC-USP. Minerva Pesq Tecnol 2009;6(1): 23-30.

18. Martins JG. Aprendizagem baseada em problemas aplicada em ambiente virtual de aprendizagem. Tese (doutorado) Universidade Federal de Santa Catarina. 2002.

Correspondência para:

Profa. Dra. Sofia Takeda Uemura

e-mail: sofia_uemura@uniararas.br

Av. Dr. Maximiliano Baruto, 500

Jd. Universitário

13607-339 Araras/SP 